\title{
Ser mujer y reducción de jornada en Galicia: Aportes del análisis del discurso crítico desde una mirada ocupacional
}

\author{
Rocío Ferreira-Marante ${ }^{1}$ y Silvia Veiga-Seijo ${ }^{1}$
}

1Universidade da Coruña, España | rocio.ferreira.marate@gmail.com; silvia.veiga.seijo@udc.es

| https://orcid.org/0000-0003-1872-4608; http://orcid.org/0000-0001-9309-2941

Resumen: Introducción: En España, la reducción de jornada es un derecho individual de las personas trabajadoras. Los motivos para solicitar dicha reducción están asociados a los cuidados, los cuales son asumidos y asignados principalmente a las mujeres.; Objetivos: Explorar el fenómeno de la reducción de jornada laboral en mujeres, dentro de la Comunidad Autónoma de Galicia, mediante el análisis de diversos medios de comunicación desde una perspectiva ocupaciona.I; Métodos: Se utilizó el análisis del discurso crítico como enfoque teórico-metodológico desde una perspectiva feminista y ocupacional. Esta metodología permite examinar cómo los discursos y el lenguaje transmitidos en los medios de comunicación dan forma y contribuyen en la construcción del imaginario social sobre el fenómeno en estudio. Se utilizó Factiva como base de datos para la búsqueda. Se establecieron palabras clave, una estrategia de búsqueda y criterios de selección. El proceso de selección de las noticias fue realizado de forma conjunta. Se ha iniciado un análisis temático, que se encuentra en proceso.; Resultados: Se incluyeron 61 noticias de periódico. 5 categorías temáticas preliminares fueron creadas a partir del análisis, que versan sobre: despido y procesos judiciales, políticas públicas asociadas a la reducción de jornada, violencia institucional, reducción de jornada desde enfoque positivo, y experiencias de reducción de jornada vinculada a los hombres.; Conclusiones: En investigación cualitativa, el análisis del discurso crítico parece ser una herramienta útil para el análisis del contenido transmitido en noticias de periódico digitales. Ello permite (re)pensar las relaciones de poder vinculadas al género que se producen cuando las mujeres participan en ocupaciones, como es en el área laboral.

Palabras clave: Análisis del Discurso Crítico; Reducción de Jornada; Género; Derechos; Terapia Ocupacional.

Being a Woman and Reduction of Working Time in Galicia: Contributions of the Critical Discourse Analysis from an Occupational Perspective

Abstract: Introduction: In Spain, the reduction of working time is an individual right of workers. The reasons to apply for a reduction of working time are associated with the care of others, which are assumed and assigned to women.; Goals: To explore the reduction of working time in women's phenomenon, in the community of Galicia, through the analysis of diverse means of communication or media from an occupational perspective.; Methods: a critical discourse analysis was conducted as a theoretical-methodological approach from a feminist and occupational perspective. This methodology allows us to examine how the discourses and language informed in media creates the social imaginary about the study topic. We employed Factiva as a database for the search. Keywords, a strategy search and selection criteria were established by authors. The selection process was conducted together by authors. The thematic analysis was started and it is in process.; Results: 61 digital news articles were included. 5 preliminary thematic categories were created: firing and judicial processes, public policies associated with reduction of working time, institutional violence, reduction of working time from a positive approach, and experiences of reducing working time linked to men.; Conclusions: In qualitative research, the critical discourse analysis seems to be a useful tool to analyse the content of digital news articles. This lets (re)thinking of the power relations associated with gender, that are produced when women participate in occupations, as for instance the labor sphere.

Keywords: Critical Discourse Analysis; Reduction of Working Time; Gender; Rights; Occupational Therapy.

\section{Introducción}


Cuidar y ser cuidadas, algo imprescindible para la supervivencia y la vida, se escribe y vivencia en femenino. Detrás del cuidado se han encontrado de forma histórica las mujeres, no por elección, sino por la asignación sexual hegemónica de los roles productivos y reproductivos (Pazos-Morán, 2013; Mayobre \& Vázquez, 2015). Los cuidados son tiempo de trabajo no remunerado, lo cual queda fuera de las lógicas neoliberales, ya que no inciden de forma directa en la acumulación de bienes. En este sentido, el bajo gasto público destinado al cuidado es compatible con un modelo de estado de bienestar en el que las mujeres realizan el trabajo no remunerado, aunque esa asociación se está demostrando insostenible (Díaz-Gorfinkiel \& Martínez-Buján, 2018). En este sentido, los cambios producidos en el mercado laboral en los últimos años, marcados por la inestabilidad financiera, altos niveles de desempleo y envejecimiento de la población activa, han orientado las políticas públicas hacia la flexibilización laboral y la precariedad (Dennhardt \& Rudman, 2012).

Este escenario ha dado lugar a la necesidad de conciliar la vida personal, familiar y laboral de la persona trabajadora; siendo la reducción de jornada una posibilidad. En España, la Ley del Estatuto de los Trabajadores (Ministerio de Empleo y Seguridad Social, 2015), en su artículo 37, presenta la reducción de jornada como un "derecho individual de los trabajadores, hombres o mujeres". Este artículo expone que la reducción de jornada puede ser solicitada por las personas trabajadoras por motivos de: nacimiento o adopción, por cuidado de personas menores de 12 años, personas con discapacidad que no desempeñe actividad retribuida, cuidado directo de un familiar, hospitalización, cuidado continuo y permanente, o presentar la consideración de víctima de violencia de género o de terrorismo. En función del caso, estas condiciones pueden suponer una disminución proporcional del salario o incluso la flexibilización horaria. No obstante, los criterios para solicitar dichas jornadas laborales dependen de los convenios colectivos o incluso de las políticas de las empresas, lo cual puede generar gran heterogeneidad en los derechos de las personas trabajadoras (Ministerio de Empleo y Seguridad Social, 2015). En este trabajo, se problematiza sobre la naturaleza de estos motivos, pues éstos están relacionados con los cuidados, los cuales en su mayoría son asumidos y asignados a mujeres. Además, la disminución de horas asalariadas a causa de la reducción de jornada laboral conlleva asumir tiempo de cuidados, que son un trabajo invisible.

En España, las mujeres son las que trabajan de forma mayoritaria a tiempo parcial (INE, 2020), lo que indica cómo deben ocupar trabajos con características más precarias, manifestando así la falta de apoyo público para favorecer la conciliación y los cuidados. Por otro lado, en Galicia, un $85,9 \%$ de mujeres abandonaron su trabajo durante un mes seguido para dedicarse al cuidado de hijos/as (IGE, 2019). Ambos datos implican que las mujeres son las principales solicitantes de la reducción de la jornada laboral, lo que puede conllevar un gran impacto sociolaboral en sus vidas: disminución de ingresos, menor poder de negociación y pensiones más bajas (Castro-García, 2017).

A nivel legislativo, se han promulgado diferentes normativas en el marco nacional e internacional (Organización de las Naciones Unidas, 2015), que pretenden incorporar de forma transversal políticas de igualdad en la agenda política, encaminadas a alcanzar la equidad social. Por ejemplo, en Galicia existe el Plan Gallego de Conciliación y Corresponsabilidad (Xunta de Galicia, 2018) para favorecer la compatibilización de cuidados y trabajo. No obstante, cabe reflexionar si estas medidas suponen un impacto real en la vida diaria de las mujeres (Lombardo \& León, 2014).

Desde una mirada de la Terapia Ocupacional, el hecho de que la reducción de jornada sea solicitada principalmente por mujeres, puede generar desigualdades en sus derechos laborales. Además, la motivación de una reducción de jornada conlleva el desempeño de diversidad de cuidados, trabajo no remunerado que invisibiliza a las mujeres. Este fenómeno ha sido poco explorado desde una mirada ocupacional y con perspectiva feminista, pudiendo deberse a que son temáticas que quedan fuera de las lógicas dominantes de la Terapia Ocupacional (Kantartzis \& Molineux, 2011).

Así, la pregunta de investigación de este trabajo es: ¿cómo es la visión que se transmite sobre la reducción de jornada laboral desde los medios de comunicación? ¿cuál es la 
construcción social que se genera a partir de la visión transmitida? ¿qué implicaciones presenta esto para la vida de mujeres que experimentan una reducción de jornada laboral?

\section{Objetivo}

El objetivo principal de esta investigación es explorar el fenómeno de la reducción de jornada laboral en mujeres, dentro de la Comunidad Autónoma de Galicia, mediante el análisis de diversos medios de comunicación desde una perspectiva ocupacional.

\section{Metodología}

\subsection{El Análisis del Discurso Crítico como Enfoque Metodológico}

La metodología empleada fue el Análisis del Discurso Crítico, el cual permite examinar cómo el lenguaje transmitido en los medios de comunicación dan forma y contribuyen en la construcción del imaginario social sobre un determinado fenómeno, como puede ser en este caso la reducción de jornada en mujeres (Greckhamer \& Cilesiz, 2014; Laliberte, 2005; Laliberte Rudman \& Dennhardt, 2015).

Esta metodología fue empleada recientemente en diversos artículos dentro del campo de la Terapia Ocupacional desde una perspectiva crítica, en temáticas como la jubilación (Laliberte et al., 2009) o procesos migratorios en población infantil en Suecia (Opland et al., 2015). Esta metodología permite comprender la "ocupación como situada en condiciones políticas, económicas, culturales, de género, $u$ otro tipo de condiciones sociales (...) y aumentar la comprensión de cómo las injusticias y desigualdades con respecto a la ocupación y las posibilidades de participar en la ocupación son formadas y perpetradas mediante los discursos" (Laliberte Rudman \& Dennhardt, 2015, p.148). Así, este análisis permite determinar cómo las posibilidades para la ocupación en la vida diaria son socialmente construidas (Laliberte et al., 2009) y situar los significados que las personas otorgan a sus ocupaciones cotidianas dentro de un contexto concreto (Laliberte Rudman \& Dennhardt, 2015, p.148; Laliberte et al., 2009).

Además, Opland et al., (2015) exponen que el análisis del discurso crítico permiten entender cómo los medios de comunicación transmiten discursos dominantes que pueden influir en cómo las personas orquestan sus ocupaciones, es decir, lo que eligen "hacer" o "no hacer". Así, esta metodología permite situarse en medio de las relaciones de poder presentes en los discursos (Matthews, 2019) y cómo estos dan forma a las oportunidades cotidianas.

\subsection{Proceso de Búsqueda}

Para el proceso de búsqueda se ha utilizado Factiva, una base de datos electrónica que recopila información de fuentes como periódicos, webs, blogs y multimedia. Se seleccionó esta fuente ya que el interés de la investigación era conocer la visión que se transmite en los medios de comunicación sobre el tema de este trabajo. De esta forma, se pretendía profundizar en los imaginarios que los medios crean y difunden.

La búsqueda fue realizada el 2 de mayo de 2020 de forma conjunta por las autoras.

Las palabras clave para realizar la búsqueda fueron: "reducción de jornada", "mujer", y "trabajo", los cuales fueron combinados con los operadores booleanos "OR" y "AND".

La estrategia de búsqueda llevada a cabo en Factiva fue ("reducción de jornada") AND ("mujer" OR "género" OR "feminismo") AND ("trabajo" or "empleo").

La Tabla 1 presenta los criterios de selección establecidos para la búsqueda: 
Vol. 9 | Investigación Cualitativa en Ciencias Sociales: Avances y Desafíos

Tabla 1. Criterios de selección de los artículos

\begin{tabular}{lc}
\multicolumn{1}{c}{ Criterios } & Características \\
\hline $\begin{array}{l}\text { Año de } \\
\text { publicación }\end{array}$ & $\begin{array}{r}\text { No se establecieron límites temporales debido a que el interés era conocer de } \\
\text { forma amplia la temática de estudio }\end{array}$ \\
$\begin{array}{l}\text { Fuentes } \\
\text { informativas }\end{array}$ & Se incluyeron todos los tipos de fuente informativas disponibles en Factiva \\
Idioma & Español, inglés y portugués \\
Temática & $\begin{array}{c}\text { Centradas en el fenómeno de la reducción de jornada en mujeres. } \\
\text { Contenido referente a la Comunidad Autónoma de Galicia. }\end{array}$ \\
\end{tabular}

\subsubsection{Proceso de Selección de los Artículos}

El número de resultados recopilados en Factiva fueron 184. Tras la eliminación de duplicados $(n=86)$, el número de documentos a revisar fue de 98 . Este proceso de búsqueda y selección de artículos fue realizado conjuntamente por ambas autoras. Tras eliminar los duplicados, ambas autoras leyeron el título y el cuerpo del artículo a texto completo de los 98 documentos. Este análisis conjunto favoreció generar debate crítico sobre el contenido de los artículos entre ambas.

En esta primera fase de análisis se excluyeron 37 artículos, porque la temática no se ajustaba a los objetivos de la presente investigación. Tras esto, se incluyeron un total de 61 noticias de periódico, que se encuentran en proceso de análisis.

\subsection{Proceso de Análisis Preliminar}

Se utilizó el enfoque del análisis del discurso crítico (Laliberte Rudman, 2005) el cual permitió analizar los artículos de periódico incluidos en este trabajo. Las autoras han seleccionado un análisis temático (Braun \& Clarke, 2006) para presentar los temas en el apartado de Resultados. Además, para este análisis, las autoras han empleado una perspectiva feminista.

Así, de forma conjunta, las autoras han leído las noticias de periódico y han realizado una codificación preliminar de la información de las mismas. Con el fin de facilitar el proceso de análisis, hicieron uso de una hoja de Excel en línea, en la que desarrollaron las siguientes variables: título de las noticias, año, autoría, fuente (periódico), lugar de publicación de la noticia, idioma y motivo de inclusión de la noticia. Esto ayudó a ambas autoras a organizar la información de los artículos e iniciar proceso de análisis preliminar. Tras esto, organizaron las noticias de periódico por similitud de contenido, generando unos temas tentativos explicados en los resultados. Para ello, las autoras establecieron categorías por colores para categorizar en esta primera fase las noticias según su contenido principal. Así, a través de los colores fue fácilmente identificable qué noticias presentan temas en común y generar un mapa visual que ha permitido avanzar en el análisis. Esto ha sido especialmente relevante en el actual contexto de pandemia por COVID-19, ya que las autoras han trabajado y compartido desde el inicio por vía telemática.

\subsection{Aspectos Éticos}

En este trabajo no se ha solicitado revisión por parte de un Comité de Ética ya que la recopilación de la información proviene de fuentes de dominio público. No se han incluido personas como informantes para responder al objetivo de investigación. 


\subsection{Rigor y Transparencia}

Para llevar a cabo el plan de análisis, se ha tenido en consideración los criterios de rigor y de transparencia propios del análisis del discurso crítico como sostienen Greckhamer \& Cilesi (2014).

\section{Resultados}

Los resultados preliminares de este trabajo presentan 5 categorías interrelacionadas que explican el fenómeno de ser mujer y la reducción de jornada en Galicia.

El año de publicación de las revistas se extiende entre 2007-2020. La Figura 1 presenta un análisis del número de artículos incluidos por año de publicación.

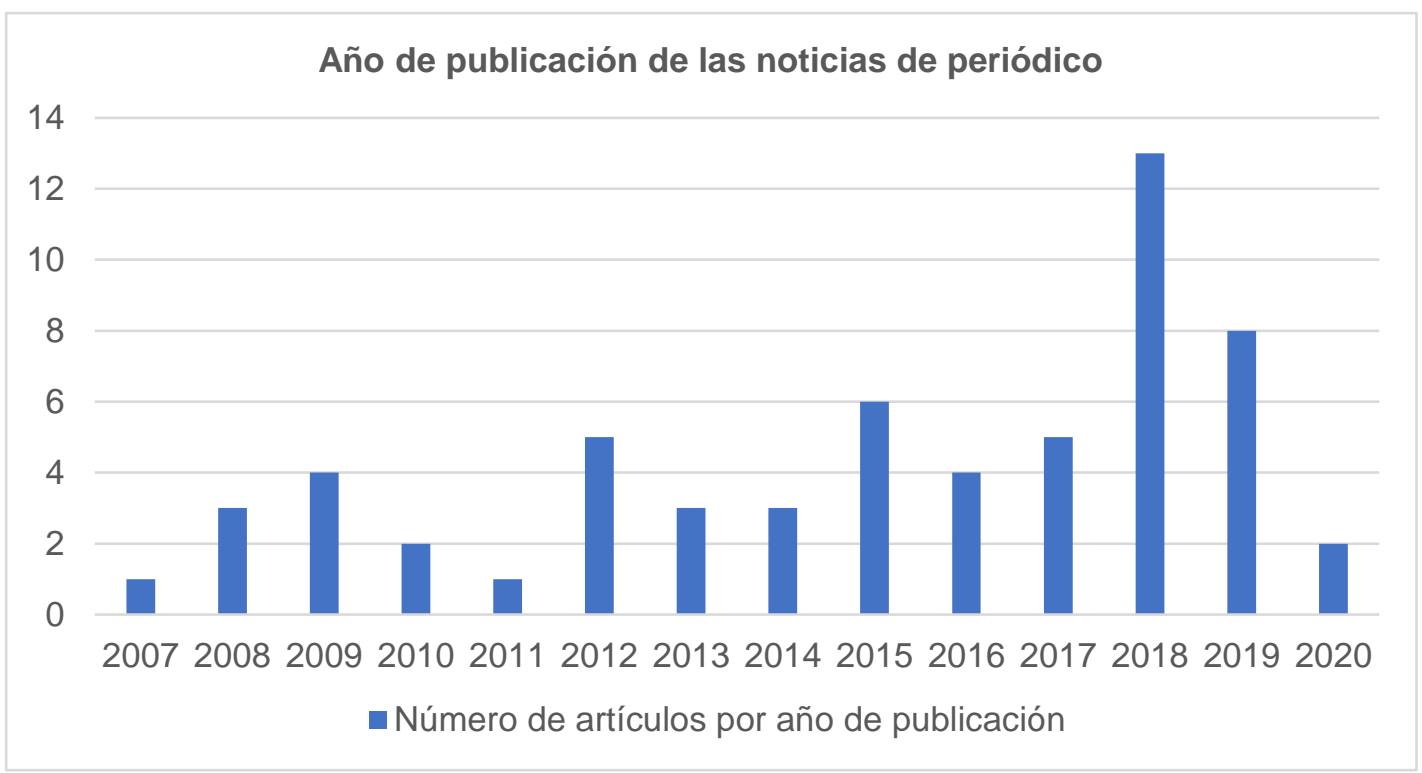

Fig. 1. Número de artículos incluidos por año de publicación

Todos los artículos incluidos en este estudio son noticias de periódico electrónicas. Una de ellas presenta contenido audiovisual. Se han identificado 8 diarios en los que han sido publicadas las noticias: Europa Press, La Voz de Galicia, Faro de Vigo, El Progreso, ABC, El País , El Confidencial y El Español. Europa Press $(n=22)$ y La Voz de Galicia $(n=19)$ han sido los principales diarios de los que se han recopilado los artículos. El idioma de todas las noticias es español.

Las principales categorías temáticas extraídas del análisis son presentadas a continuación. Por un lado, A) las noticias exponen casos de despido vividos por mujeres que solicitaron la reducción de jornada, así como los procesos judiciales asociados a los mismos. En estos casos, las mujeres informan cómo la negación de la reducción de jornada presenta un impacto en sus vidas y por tanto en su bienestar. Por ejemplo, una noticia presenta el caso de una mujer a la que se le niega el derecho a conciliar la lactancia natural de forma íntima (Cela, 2018). Así mismo, en algunas noticias (B) se aprecia violencia institucional debido a la ausencia o negación de medidas que favorezcan la reducción de jornada. 
Otro grupo de noticias (C), se centran en informar sobre políticas públicas, concretamente medidas a implementar para favorecer la conciliación y la reducción de jornada. No obstante, algunas noticias presentan discursos que llaman a la necesidad de un cambio en las políticas sociales relacionadas con la reducción de jornada. Entre estas noticias, diferentes organizaciones sindicales reclaman mejoras en los derechos laborales.

Por otro lado, dos noticias (D) presentan el fenómeno de la reducción de jornada experimentada por mujeres desde un enfoque positivo. Por último, los artículos incluidos (E) también presentan experiencias de reducción de jornada vinculadas a los hombres.

\section{Conclusiones}

En esta investigación, el análisis del discurso crítico parece ser una herramienta útil para visibilizar los discursos e imaginarios presentes en los medios de comunicación digitales sobre la reducción de jornada, desde una perspectiva ocupacional y feminista. Este enfoque teórico-metodológico permite adentrarse en las relaciones de poder y estructuras políticas presentes en los poderes fácticos, que impactan y/o determinan las elecciones y oportunidades ocupacionales; como se percibe en este estudio en el caso de las mujeres. Por tanto, el análisis del discurso crítico puede permitir generar conocimiento sobre una cuestión social desde una investigación cualitativa crítica, incluso en contextos de pandemia en los que la presencialidad puede estar limitada por restricciones y medidas de seguridad sanitarias.

Así, el análisis de los discursos permitió analizar el impacto que las noticias de periódico tienen en la sociedad y qué asociaciones se realizan en base al género, como por ejemplo, dar por sentado que la reducción de jornada es una cuestión femenina. Estos discursos generan y construyen a su vez la realidad cotidiana de las personas, fenómeno de interés en Terapia Ocupacional.

Además, esta metodología fue valiosa para generar la primera fase de análisis mediante una investigación a distancia-online. El hecho de realizar la búsqueda en Factivia al inicio de la pandemia, implica que las noticias generadas a posteriori vinculadas al COVID-19 no han sido incluidas en este trabajo. Por ello, sería interesante ampliar la búsqueda para incluir nuevos escenarios relacionados con el fenómeno de estudio, que actualmente se relacionan con los cambios en las políticas laborales por COVID-19 y las múltiples circunstancias vitales de las mujeres.

Por último, esta experiencia proporciona orientaciones teórico-metodológicas para articular una futura investigación. Así, el presente trabajo podría ser considerado una primera fase que guíe una investigación más amplia, mediante el diseño de un estudio cualitativo en el que se utilicen diversas técnicas de generación de datos cualitativas y en el que mujeres en situación de reducción de jornada sean las protagonistas.

\section{Agradecimientos}

Las autoras de esta investigación agradecen al equipo revisor sus aportaciones, ya que han contribuido a mejorar la calidad de este trabajo.

\section{Referencias}

Braun, V., \& Clarke, V. (2006). Using thematic analysis in psychology. Qualitative Research in Psychology, 3(2), 77-101. https://doi.org/10.1191/1478088706qp063oa

Castro-García, C. (2017). Políticas de Igualdad. Permisos por nacimiento transformación de roles de género. Los libros de la Catarata.

Cela, D. (21 de septiembre de 2018). «Dejo el camino abierto para otras madres». La Voz de Galicia. https://www.lavozdegalicia.es/noticia/lugo/lugo/2018/09/20/dejo-camino-abiertomadres/0003_201809G20P29992.htm 
Dennhardt, S., \& Rudman, D. L. (2012). When Occupation goes 'Wrong': A Critical Reflection on Risk Discourses and their Relevance in Shaping Occupation. En Wiley Online Books. Occupational Science: Society, Inclusion, Participation (pp. 117-133). https://doi.org/doi:10.1002/9781118281581.ch9

Díaz-Gorfinkiel, M., \& Martínez-Buján, R. (2018). Mujeres migrantes y trabajos de cuidados: transformaciones del sector doméstico en España. Panorama SOCIAL, (27), 105-118.

Greckhamer, T., \& Cilesiz, S. (2014). Rigor, transparency, evidence, and representation in discourse analysis: Challenges and recommendations. International Journal of Qualitative Methods, 13, 422-443. https://doi.org/10.1177/160940691401300123

IGE. (2019). Enquisa de poboación activa. Módulo sobre conciliación entre vida laboral e familiar.

INE. (2020). Encuesta de Población Activa (EPA). Variables de submuestra. Año 2019.

Kantartzis, S., \& Molineux, M. (2011). The Influence of Western Society's Construction of a Healthy Daily Life on the Conceptualisation of Occupation. Journal of Occupational Science, 18(1), 62-80. https://doi.org/10.1080/14427591.2011.566917

Laliberte Rudman, D. (2005). Understanding political influences on occupational possibilities: An analysis of newspaper constructions of retirement. Journal of Occupational Science, 12(3), 149-160. https://doi.org/10.1080/14427591.2005.9686558

Laliberte Rudman, D, Huot, S., \& Dennhardt, S. (2009). Shaping ideal places for retirement: Occupational possibilities within contemporary media. Journal of Occupational Science, 16(1), 18-24. https://doi.org/10.1080/14427591.2009.968663

Laliberte \& Denhardt (2014). Critical discourse analysis. Opening possibilities through deconstruction. Nayar \& Stanley. En Qualitative research methodologies for occupational science and therapy (pp. 137-154). Routledge.

Lombardo, E., \& León, M. (2014). Políticas de igualdad de género y sociales en España: origen, desarrollo y desmantelamiento en un contexto de crisis económica. Investigaciones Feministas, 5, 13-35. https://doi.org/10.5209/rev_INFE.2014.v5.47986

Matthews, J. (2019). Cultural otherness and disaster news: The influence of western discourses on Japan in US and UK news coverage of the 2011 Great East Japan Disaster. International Communication Gazette, 81(4), 372-392. https://doi.org/10.1177/1748048518774982

Mayobre, P., \& Vázquez, Iria. (2015). Cuidar cuesta: Un análisis del cuidado desde la perspectiva de género. Revista Española de Investigaciones Sociológicas, 151, 83-100. http://dx.doi.org/10.5477/cis/reis.151.83

Opland Stenersen, A., Laliberte Rudman, D., \& Raanaas, R. K. (2016). Shaping Occupational Possibilities for Norwegian Immigrant Children: A Critical Discourse Analysis. Journal of Occupational Science, 23(1), 17-32. https://doi.org/10.1080/14427591.2015.1070783

Organización de las Naciones Unidas. (2015). Transformar nuestro mundo: la Agenda 2030 para el Desarrollo Sostenible.

Pazos-Morán, M. (2013). Bases para una economía política feminista. En Desiguales por Ley. Las políticas públicas contra la desigualdad de género (pp. 33-70). Los libros de la Catarata.

Real Decreto Legislativo 2/2015, de 23 de octubre, por el que se aprueba el texto refundido de la Ley del Estatuto de los Trabajadores. Ministerio de Empleo y Seguridad Social (2015). 24 de octubre de 2015, núm. 255, pp. 1-86.

Xunta de Galicia. (2018). Plan Galego de Conciliación e Corresponsabilidade 20182021.http://igualdade.xunta.gal/sites/default/files/files/documentos/plan_galego_cc_2018040 6.pdf 